This is the post peer-review accepted manuscript of:

L. Perilli, M. Pizzotti, L. Perugini, E. Franchi Scarselli and R. Canegallo, "PV Cell Characteristic Extraction to Verify Power Transfer Efficiency in Indoor Harvesting System," in: 2018 IEEE 23rd International Workshop on Computer Aided Modeling and Design of Communication Links and Networks (CAMAD), Barcelona, 2018, pp. 16.

The published version is available online at:

https://doi.org/10.1109/CAMAD.2018.8515002

(C) 2018 IEEE. Personal use of this material is permitted. Permission from IEEE must be obtained for all other uses, in any current or future media, including reprinting/republishing this material for advertising or promotional purposes, creating new collective works, for resale or redistribution to servers or lists, or reuse of any copyrighted component of this work in other works. 


\section{PV Cell Characteristic Extraction to Verify Power Transfer Efficiency in Indoor Harvesting System}

\author{
L. Perilli, M. Pizzotti, L. Perugini, E. Franchi Scarselli \\ ARCES - DEI \\ University of Bologna \\ Bologna, Italy \\ \{luca.perilli, matteo.pizzotti, eleonora.franchi\}@unibo.it
}

\author{
R. Canegallo \\ STMicroelectronics \\ Agrate Brianza, Italy \\ roberto.canegallo@st.com
}

\begin{abstract}
A method is proposed to verify the efficiency of low-power harvesting systems based on Photovoltaic (PV) cells for indoor applications and a Fractional Open-Circuit Voltage (FOCV) technique to track the Maximum Power Point (MPP). It relies on an algorithm to reconstruct the $P V$ cell Power versus Voltage $(\mathrm{P}-\mathrm{V})$ characteristic measuring the open circuit voltage and the voltage/current operating point but not the short-circuit current as required by state-of-the-art algorithms. This way the characteristic is reconstructed starting from the two values corresponding to standard operation modes of dc-dc converters implementing the FOCV Maximum Power Point Tracking (MPPT) technique. The method is applied to a prototype system: an external board is connected between the transducer and the dc-de converter to measure the open circuit voltage and the voltage/current operating values. Experimental comparisons between the reconstructed and the measured $P-V$ characteristics validate the reconstruction algorithm. Experimental results show the method is able to clearly identify the error between the transducer operating point and the one corresponding to the maximum power transfer, whilst also suggesting corrective action on the programmable factor of the FOCV technique. The proposed technique therefore provides a possible way of estimating MPPT efficiency without sampling the full P-V characteristic.
\end{abstract}

Keywords-DC-DC power conversion, energy harvesting, low power electronics, maximum power point trackers, photovoltaic cells

\section{INTRODUCTION}

In many low-power energy harvesting applications such as Photovoltaic (PV) panels or Thermoelectric Generators (TEG), with power available in the order of hundreds of microwatts, load matching is required to achieve maximum power transfer. Energy harvesting applications have limited bandwidth requirements and can usually enter low-power states for long periods of time; in order to harvest the maximum amount of power from energy sources when the transducer power curve varies with the source intensity and the load varies greatly with the system state, a dc-dc converter followed by an energy tank element (Fig. 1(a)) is therefore required to store surplus energy extracted from the source and make it available for the load in periods when harvested energy is lower than system consumption (such as night-time hours for PV cells). The

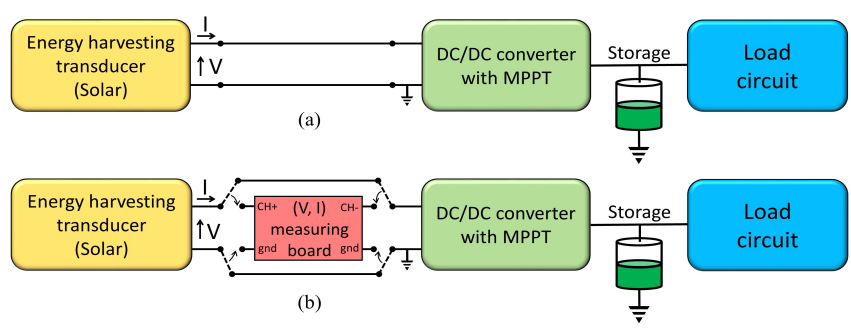

Fig. 1. Main blocks composing a generic energy harvesting autonomous system. Standard (a) and proposed system (b) which allows for insertion of a measuring board between the transducer and the dc-dc converter.

energy storage element may be a rechargeable battery or a super-capacitor.

In systems with de energy sources whose output power depends on converter input impedance, the dc-dc converter element is composed of two main blocks. The first is the conversion block, which transfers the harvested energy between the transducer and supply nodes (usually kept at different voltages). The second block is the Maximum Power Point Tracking (MPPT) unit that keeps the dc-dc converter input voltage at the optimum value for the given environmental conditions in order to optimize transducer power transfer efficiency.

Many MPPT techniques have been proposed [1]. In high power systems, such as outdoor PV panels, where dc-dc converter power consumption contribution is negligible, closed looped and computational intensive MPPT algorithms are used. In low-power applications, such as those based on indoor PV panels or thermoelectric modules, dc-dc converters with open loop MPPT techniques such as Fractional Open-Circuit Voltage (FOCV) are used [2]. The FOCV algorithm assumes a simplified linear dependence between the voltage corresponding to the Maximum Power Point (MPP) and the open-circuit voltage $\left(V_{O C}\right)$ of the transducer. Whereas $V_{O C}$ is periodically read to track variations in operating conditions, a constant multiplying factor $(k)$ is assumed that is programmed once and for all at the dc-dc converter input, depending on the application. Incorrect choice of the parameter $k$ results in a loss of efficiency of the harvesting system, especially since its value is actually slightly dependent on the source intensity [3].

In this paper we propose a method whereby the efficiency of the FOCV-MPPT algorithm in a low-power harvesting 
system based on indoor PV panels may be verified. The proposed system relies on an algorithm to reconstruct the complete PV cell Power versus Voltage (P-V) characteristic without needing the value of panel short-circuit current, as in [4][5], but only the values of voltages and currents at the transducer output during normal operation of a system implementing the FOCV-MPPT technique. This way, verifying MPPT efficiency does not require dedicated power-consuming circuitry to be added to the original harvesting system, nor power loss due to short-circuit current measurement, but only the possibility of inserting an external measuring unit between the panel and the dc-dc converter. With reference to Fig. 1(b), during normal operation the transducer is directly connected at the dc-dc converter input, while during the debug phase the external measuring board is connected in series between the transducers and the dc-dc converter input and allows one to measure voltage and current $(V, I)$ values minimizing voltage drop.

In section II the system specifications, the drawbacks of existing low-power FOCV solutions and the proposed solution are presented. The indoor PV cell model and the algorithm to reconstruct the complete panel characteristic are discussed in section III. Experimental results are presented in section IV: we discuss the performance of the algorithm to reconstruct the P-V characteristic, and then its ability to estimate MPPT efficiency. Conclusions are drawn in section $\mathrm{V}$.

\section{PV-BASED INDOOR HARVESTING SYSTEM}

\section{A. System Specification}

The proposed method aims to verify the efficiency of lowpower harvesting systems based on PV cells for indoor applications; the efficiency is verified only during the installation or debugging phases and not run-time as proposed in other works, for example in [6].

The method is applied to indoor PV harvesting systems in which the light intensity levels are usually rated in terms of photometric units (lx); as an example, in the case of standard global AM 1.5 spectrum, an illuminance of 500 lx corresponds to an irradiance of $500 \mu \mathrm{W} / \mathrm{cm} 2$ [7]. The indoor illumination levels range from 100 lx to 1000 lx: a range of $100-300 \mathrm{~lx}$ is typical of home lighting, the range 400-800 $\mathrm{lx}$ is typical of office and meeting rooms, while near the window the illuminance level will reach $1000 \mathrm{~lx}$. Moreover, in indoor environments the temperature excursions are modest so the temperature dependence of the PV cell characteristic can be ignored.

\section{B. FOCV-MPPT Algorithms}

MPPT algorithms are used to keep the load seen by the transducer near the optimum value. This is necessary because the P-V curve of transducers has a single maximum at a specific voltage and decreases to zero at higher and lower voltages. As an example, Fig. 2 shows typical P-V and current versus voltage (I-V) curves of a PV panel for a fixed illuminance level.

FOCV algorithm is used in dc-dc converters for low energy applications. It is based on the assumption that, ignoring

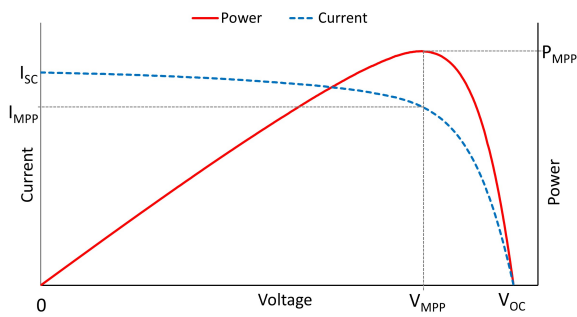

Fig 2. Photovoltaic cell P-V and I-V curves. Voltage $V_{M P P}$ and current $I_{M P P}$ correspond to maximum power transfer $P_{M P P}$.

temperature dependence, $V_{M P P} \sim k * V_{O C}$, where $V_{M P P}$ is the voltage corresponding to maximum power transfer $P_{M P P}$ and $V_{O C}$ is the open circuit voltage of the transducer. This assumption is valid for PV cell [2] and TEG models [8]. According to this technique, as sketched in Fig. 3, during the conversion phase $\left(T_{\text {TRACKING }}\right)$, the dc-dc converter forces the transducers to operate at the working point:

$$
V_{M}=k * V_{O C}
$$

where the value of the multiplying factor $k$ is chosen according to the physical transducer characteristics (typical values are $k \sim$ 0.5 for TEG [8] and $k \sim 0.7-0.8$ for PV cells [2] [3]) and is fixed for an application typically using voltage references or voltage dividers at dc-dc converter input. At regular intervals the MPPT logic embedded in the dc-dc converter disconnects itself for a short period of time and enters the measuring phase $\left(T_{\text {SAMPLE }}\right)$ where the transducers' output voltage $V_{O C}$ in an opencircuit state is measured to update the voltage operating point following (1).

Techniques are proposed in order to reduce the $V_{O C}$ sampling period and the momentary power loss when the load is disconnected from the transducer [2][8].

\section{System Implementation}

In order to design a system based on indoor PV cells and an FOCV-MPPT technique, a developer needs to set the $k$ factor according to the panel characteristics. Two voltage values are needed: the value in open-circuit $\left(V_{O C}\right)$ and in maximum power transfer $\left(V_{M P P}\right)$ operating points at the same illuminance level. If not available from the transducer datasheet, $V_{O C}$ can easily be measured by disconnecting the panel, while $V_{M P P}$ can only be measured by sampling the full P-V (or I-V) characteristic, as shown in Fig. 2, changing the panel load until the maximum output power is obtained. Given these measurements, $k=V_{M P P}$ / $V_{O C}$ can be set in the dc-dc converter and in common implementations this value is considered accurate for all

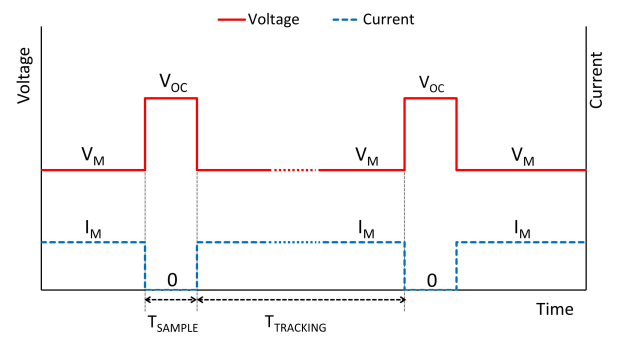

Fig. 3. Sketch, not in scale, of voltage (top) and current (bottom) waveforms at the transducer output of the harvesting system based on the FOCV-MPPT technique. 
illuminance values. However, the $k$ factor is a function of panel fabrication process, illuminance and temperature values in operating conditions. As discussed in section II.A, in indoor applications, the temperature dependence can be ignored since the temperature variations are limited. On the contrary, illuminance levels in indoor environments range from $100 \mathrm{~lx}$ to $1000 \mathrm{~lx}$. In order to define the value of the $k$ factor optimizing power transfer of the specific panel under analysis, complete P$\mathrm{V}$ curve characterization would therefore be required for a number of illuminance levels, but PV panel manufacturers often do not include these characterizations in datasheets and specific equipment is required for full panel characterization on site.

To overcome the problem, analytic-based MPPT techniques are proposed: from experimental measurement of $V_{O C}$ and short-circuit current $\left(I_{S C}\right)$, techniques for analytical calculation of the MPP of a photovoltaic array are suggested in [4][5].

Implementing these MPPT techniques in a dc-dc converter still requires integration of additional power-consuming circuitry to measure $I_{S C}$ and to compute $V_{M P P}$, which is not well suited to low-power applications.

Unlike the previous technique, we propose a method that allows reconstruction of the $\mathrm{P}-\mathrm{V}$ curve without requiring measurement of $I_{S C}$, but only the values $\left(V_{M}, I_{M}\right)$ and $\left(V_{O C}, 0\right)$ corresponding to normal FOCV-MPPT operation, as sketched in Fig. 3. The only additions to the original harvesting system are a few passive components (switch and connector) to allow insertion of a measuring board between the transducer and the dc-dc converter (Fig. 1(b)) whenever the efficiency of the power transfer has to be monitored. The acquired data are sent to a PC where the reconstruction algorithm is executed. Comparison between the working point $\left(V_{M}, I_{M}\right)$ and the optimal one $\left(V_{M P P}, I_{M P P}\right)$ corresponding to maximum power $P_{M P P}$ calculated from the reconstructed characteristic gives an estimate of the efficiency of the MPPT algorithm and allows one to modify the value of the multiplying $k$ factor accordingly.

\section{ALGORITHM TO RECONSTRUCT PV CHARACTERISTIC}

\section{A. Photovoltaic Cell Model}

The PV cell model is proposed in [9] and refers to an amorphous panel, as shown in Fig. 4 that leads to (2).

$I_{G}$ is the photo-generated current proportional to light irradiation. In crystalline cells this term is independent of the operating point. In amorphous cells, such as those used in the system analyzed, there is an additional recombination current loss proportional to $I_{G}$ and dependent on the operating point, modeled with the corrective terms $\left(b_{1}, b_{2}\right)$ on $I_{G}$ : the effective photo-generated current is therefore $I_{P G}<I_{G}$. The PV panel, being composed of a series of p-n junctions, has a dark current component $I_{D}$ which must be subtracted from $I_{P G}$. In the $I_{D}$ model, $I_{0}$ is the diodes dark saturation current, $n_{s}$ the number of cells in series, $V_{T}=k^{*} T / q$ the thermal voltage and $a$ (a real number between 1 and 2) the diode non-ideality factor related to additional recombination effects. The illuminance is assumed uniform on all the $n_{s}$ cells. Along with these core parameters, additional cell losses are modeled with resistors: $R_{S H}$ is the shunt resistance related to manufacturing defect losses in the device; $R_{S}$ is the series resistance related to ohmic paths in the junction and metal contacts. This model leads to the equation:

$$
\begin{gathered}
I=I_{P G}-I_{D}-I_{S H}= \\
=I_{G}\left(1-\frac{b_{1}}{b_{2}-V-R_{S} I}\right)-I_{0}\left(e^{\frac{V+R_{S} I}{n_{s} a V_{T}}}-1\right)-\frac{V+R_{S} I}{R_{S H}} .
\end{gathered}
$$

To reconstruct the I-V curve, the value of the parameters in (2) is required. The main panel characteristics provided by PV cell manufacturers are $(V, I)$ values for one or a few illuminance levels at three operating points: open-circuit $\left(V_{O C}\right.$, $0)$, maximum power $\left(V_{M P P}, I_{M P P}\right)$ and short-circuit $\left(0, I_{S C}\right)$. It has been shown [10] that it is possible to interpolate a complete $\mathrm{I}-\mathrm{V}$ curve at a fixed illuminance from these three operating points.

An original algorithm is here proposed allowing us to reconstruct the $\mathrm{I}-\mathrm{V}(\mathrm{P}-\mathrm{V})$ curve from the only two operating points $\left(V_{M}, I_{M}\right)$ and $\left(V_{O C}, 0\right)$ corresponding to Fig. 3.

The parameters in (2) behave differently depending on the level of illuminance. A first group including $a, R_{S H}, b_{1}, b_{2}$ is mainly related to geometric and manufacturing parameters and has very limited dependency on the degree of illuminance. These parameters are therefore calculated only once by the Optimization Algorithm described in paragraph III.B. Other parameters are $I_{G}, I_{0}$ and $R_{S}$ which, on the contrary, have a deep correlation with illuminance. These parameters cannot be fixed for the system and must be recalculated under varying illuminance conditions from measured values $\left(V_{M}, I_{M}\right),\left(V_{O C}, 0\right)$ as described in paragraph III.C.

\section{B. Optimization Algorithm}

The Optimization Algorithm was developed in MATLAB ${ }^{\odot}$ and aims to find the values of $a, R_{S H}, b_{1}, b_{2}$ that best fit (2) with a set of experimental I-V panel curves. Offline full P-V panel characterization using ad-hoc testing devices or available through the manufacturer's datasheets is therefore still required in the proposed technique as in common implementation of FOCV-MPPT systems but only for a subset of illuminance curves: these parameters will allow one to reconstruct curves for all illuminance levels, as shown in section IV.C.

Starting from an initial guess as to the quadruple of parameters $a, R_{S H}, b_{1}, b_{2}$ (typical values are taken from literature [9], $b_{1}=0.1 \mathrm{~V}, b_{2}=0.9 \mathrm{~V}$, whereas $a=1.5$ and $R_{S H}=$ $2.5 \mathrm{M} \Omega$ ), the resulting quadruple is the one that minimizes the error function $e$ defined in (3) as the root mean square of the

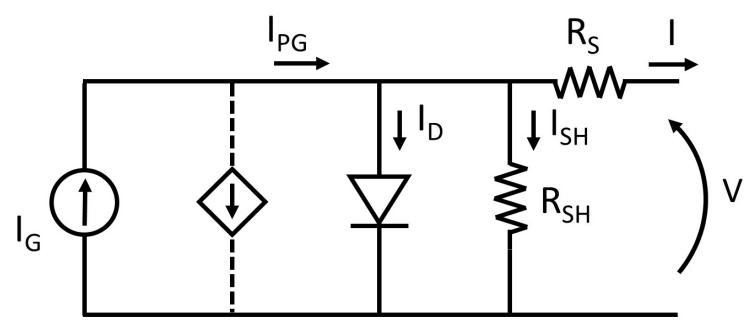

Fig. 4. Amorphous PV panel equivalent model. 
distance between all measured points $\left(I_{m, i j}\right)$ at different voltage points $\left(V_{i}\right)$ and illuminance levels $\left(L_{j}\right)$ as compared to calculated ones $\left(I_{c, i j}\right)$ using the Reconstruction Algorithm described in the following section:

$$
e=\sqrt{\sum_{V_{i}} \sum_{L_{j}}\left\|I_{m}-I_{c}\right\|_{V_{i}, L_{j}}^{2}}
$$

A minimum for that function is sought through the search method [11] offered in MATLAB ${ }^{\odot}$, a direct search method that does not use numerical or analytic gradients. It is worth noting that the obtained values of $a, R_{S H}, b_{1}, b_{2}$, are the result of the optimization phase: they act therefore as fitting parameters and may assume values that are not physically acceptable, such as the parameter $a$ slightly greater than 2 .

\section{Reconstruction Algorithm}

To solve the system for $I_{G}, I_{0}$ and $R_{s}$ three equations are needed. The first two equations are obtained by substituting in (2) the values $\left(V_{M}, I_{M}\right)$ and $\left(V_{O C}, 0\right)$ measured in the two operating phases (dc-dc conversion and open-circuit measuring) of a system implementing an FOCV-MPPT technique (see Fig. 3). To find the third equation an approximation must be introduced.

It can be shown that, in a Short-Circuit (SC) case, the derivative of (2) can be approximated as

$$
\left.\frac{d I}{d V}\right|_{S C}=-1 / R_{S H} .
$$

Approximation (4) is derived in [12] and is based on the assumption that $R_{S} I_{S C}<<a n_{S} V_{T}$. Equation (4) cannot be used in the proposed method because $I_{S C}$ is not known from measurement; the third equation is therefore derived following an approach similar to that in [12] but analyzing the derivative of (2) at the Open-Circuit (OC) point. Even if (2) is a nonlinear implicit function, its derivative can be calculated analytically using implicit partial derivatives. Calculation of the derivative in $\left(V_{O C}, 0\right)$ leads to:

$$
\left.\frac{d I}{d V}\right|_{O C}=-\frac{A}{1+R_{S} A},
$$

with

$$
A=\frac{I_{0}}{a n_{S} V_{T}} e^{\frac{V_{O C}}{a n_{S} V_{T}}}+\frac{1}{R_{S H}}+I_{G} \frac{b_{1}}{\left(b_{2}-V_{O C}\right)^{2}} .
$$

Typical values for the first term of $A$ are in the order of 1e-4 $\mathrm{S}$. The third term of $A$ can be ignored because it relates to a corrective term of $I_{G}$ and is an order of magnitude smaller than other terms (a typical value is in the order of 1e-7 S). The second term of $A$ can also be ignored in all cases where shunt losses are small (for $R_{S H}>10 \mathrm{~K} \Omega$, it is $<1 \mathrm{e}-5 \mathrm{~S}$ ). $A$ can therefore be approximated with its dominant first term. Series resistance $R_{S}$, as a parasitic parameter, is usually less than 100 $\Omega$ and this ensures that $R_{S} A<<1(1 \mathrm{e}-2)$, leading to:

$$
\left.\frac{d I}{d V}\right|_{O C} \approx-\frac{I_{0}}{a n_{s} V_{T}} e^{\frac{V_{O C}}{a n s_{s} V_{T}}}
$$

On equating this approximated (6) to (5), the third system equation is obtained:

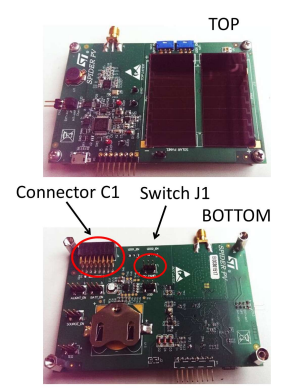

(a)

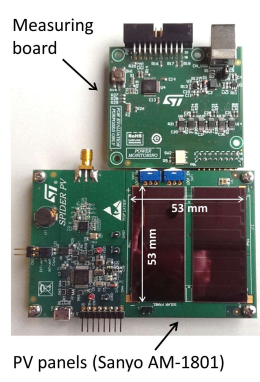

(b)
Fig. 5. (a) Top and bottom layer of the harvesting board and (b) harvesting system with measuring board mounted.

$$
\frac{A}{1+R_{S} A}=\frac{I_{0}}{a n_{s} V_{T}} e^{\frac{V_{o C}}{a n_{S} V_{T}}} .
$$

The system to compute $I_{G}, I_{0}$ and $R_{s}$ is therefore (2) calculated in $\left(V_{M}, I_{M}\right),(2)$ calculated in $\left(V_{O C}, 0\right)$ and $(7)$ :

$$
\left\{\begin{array}{c}
I_{M}=f\left(V_{M}\right) \\
0=f\left(V_{O C}\right) \\
(7)
\end{array}\right.
$$

Solving (8) for $R_{S}$ leads to an implicit nonlinear equation that cannot be solved analytically. However, this equation can be solved by numerical methods (its derivative can be calculated analytically, again with partial implicit derivatives, so Newton-Raphson [13] may be used). The $R_{S}$ value obtained can be substituted in the first two equations to solve for $I_{0}, I_{G}$.

Once all PV panel parameters are known for a given illuminance value, the I-V curve can be calculated from (2). Given that this equation is implicit and nonlinear, it must be solved numerically (again by Newton-Raphson [13]) for every point needed. From the calculated I-V curve, it is possible to obtain the P-V curve and MPP, which can be used to verify the dc-dc converter MPPT efficiency.

\section{EXPERIMENTAL RESULTS}

\section{A. Procedure to Verify the Harvesting System Efficiency}

Starting from the PV panel model, (2) and a set of panel experimental curves, the Optimization Algorithm (described in section III.B) calculates the illuminance independent parameters $\left(a, R_{S H}, b_{1}\right.$ and $\left.b_{2}\right)$ of (2). This operation need be performed only once: the obtained quadruple of parameters is then used every time the system efficiency is to be evaluated. To reconstruct the complete I-V and P-V curves of the system

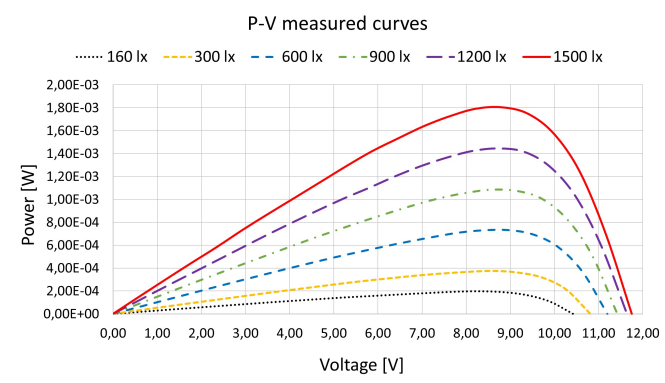

Fig. 6. Panel P-V measured curves at various different illuminance levels. 
under analysis, only the measurable values $\left(V_{M}, I_{M}\right)$ and $\left(V_{O C}\right.$, $0)$ are required by the Reconstruction Algorithm described in section III.C. The optimal operating point MPP - i.e. the voltage and current $\left(V_{M P P}, I_{M P P}\right)$ corresponding to maximum power $P_{M P P}$ - is then calculated from the reconstructed $\mathrm{P}-\mathrm{V}$ curve: the difference between the calculated $V_{M P P}$ and the voltage of the measured point $\left(V_{M}, I_{M}\right)$, i.e. the working point forced by the dc-dc converter, provides a measure of the appropriateness of the $k$ factor used by the FOCV-MPPT algorithm.

\section{B. Description of the Harvesting System Prototype}

Fig. 5 shows the implemented prototype of the energy harvesting system. The architecture follows Fig. 1: the transducer is composed of two AM-1801 [14] PV panels while module SPV1050 [15], especially devoted to ultra-low energy harvesting applications, implements the FOCV-MPPT function and integrates a buck-bust dc-dc converter. A resistor divider at the SPV1050 input allows one to program the multiplying factor $k$ value in (1). In the Sanyo AM-1801 datasheet [14], the following values are given: $V_{O C}=4.9 \mathrm{~V}$ for $V_{M P P}=3 \mathrm{~V}$ at $\mathrm{T}=$ $25^{\circ} \mathrm{C}$ and $200 \mathrm{~lx}$. An initial guess for the multiplying factor $k=$ $V_{M P P} / V_{O C}=0.61$ can therefore be made.

Any V-I measuring system that can discriminate between the two dc-dc converter operating phases sketched in Fig. 3, can be inserted as shown in Fig. 1(b). The measuring unit could be an oscilloscope but, in the system presented, a dedicated power-measuring board has been designed. Switch J1 and connector $\mathrm{C} 1$, as shown in Fig. 5(a), allow the measuring board to be inserted in series between the PV panels and the dc-dc converter, according to the connections sketched in Fig. 1(b). Fig. 5(b) shows the system with the board mounted.

\section{Validation of the P-V Reconstruction Algorithm}

The set of panel P-V curves required as input for the Optimization Algorithm was obtained by measuring the AM$1801 \mathrm{PV}$ panel at six values of illuminance level: $160 \mathrm{~lx}, 300$ lx, 600 lx, 900 lx, 1200 lx and 1500 lx. For each illuminance level, the P-V curves were plotted, as shown in Fig. 6.

To validate the Reconstruction Algorithm, the measuring board was mounted in series between the PV panels and the dcdc converter input, as in Fig. 5(b) and according to Fig. 1, and values $\left(V_{O C}, 0\right)$ and $\left(V_{M}, I_{M}\right)$ were measured for various illuminance levels, with the dc-dc converter implementing the FOCV-MPPT programmed at various different values of multiplying factor $k$. The first set of curves is reconstructed

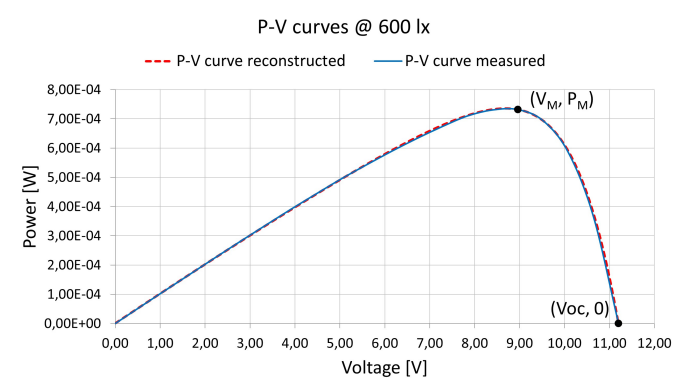

Fig. 7. Measured vs reconstructed P-V curve at $600 \mathrm{~lx} .\left(V_{M}, P_{M}\right)$ and $\left(V_{O C}, 0\right)$ sampled when $k=0.8$. from measurements taken when $k$ is programmed at 0.8 . By way of example, Fig. 7 shows the measured (solid line) versus reconstructed (dashed line) $\mathrm{P}-\mathrm{V}$ curve at an illuminance level of $600 \mathrm{~lx}$, one of the illuminance levels used for the optimization phase. The two points used for curve reconstruction are indicated: $\left(V_{M}, P_{M}=V_{M} * I_{M}\right)$ and $\left(V_{O C}, 0\right)$. The two curves are in good agreement and the error between the measured $P_{M P P}$ and the reconstructed one is $0.5 \%$. The error is defined as:

$$
P_{M P P_{-} \text {err }}=\frac{\left|P_{M P P_{-} \text {meas }}-P_{M P P_{\_} \text {reconsstr }}\right|}{P_{M P P_{-} \text {meas }}} * 100 .
$$

Fig. 8 shows the measured versus reconstructed P-V curve at an illuminance level of $450 \mathrm{~lx}$ which is not included in the set of P-V curves used for the optimization phase. In this case too, the two curves show a good agreement and the error between the measured $P_{M P P}$ point and the calculated one (defined in (9)) is $0.23 \%$.

Table I shows, for different illuminance levels, the two points used for reconstruction $\left(V_{O C}\right.$ and $\left.\left(V_{M}, P_{M}\right)\right)$, the MPP of the measured and reconstructed curves and, in the last column, the error between the maximum power points, defined in (9). The highlighted rows display the illuminance levels not included in the measured set of P-V curves used for reconstruction. In all cases tested the error between measured and calculated $P_{M P P}$ is lower than $0.55 \%$.

The difference between measured and reconstructed $V_{M P P}$ is in all cases lower than $0.3 \mathrm{~V}$; note that the $V_{M P P}$ difference results in such a low error in estimated MPP since around this point the derivative of the power curve is low.

TABLE I

MPP ERRors BetweEn MeAsured AND ReCONSTRUCTEd CURVES $\left(\left(V_{M}, I_{M}\right)\right.$ $\operatorname{AND}\left(V_{O C}, 0\right)$ SAMPLED WHEN $\left.K=0.8\right)$

\begin{tabular}{|c|c|c|c|c|c|}
\hline $\mathbf{L u x}$ & $\begin{array}{c}\mathbf{V}_{\mathbf{O C}} \\
{[\mathbf{V}]}\end{array}$ & $\mathbf{V}_{\mathbf{M}} / \mathbf{P}_{\mathbf{M}}[\mathbf{V} / \mathbf{W}]$ & $\begin{array}{c}\mathbf{V}_{\mathbf{M P P}} / \mathbf{P}_{\mathbf{M P P}}[\mathbf{V} / \mathbf{W}] \\
\text { measured }\end{array}$ & $\begin{array}{c}\mathbf{V}_{\mathbf{M P P}} / \mathbf{P}_{\mathbf{M P P}}[\mathbf{V} / \mathbf{W}] \\
\text { reconstructed }\end{array}$ & $\begin{array}{c}\mathbf{P}_{\mathbf{M P P}} \text { [\%r } \\
{[\mathbf{0}]}\end{array}$ \\
\hline $\mathbf{1 6 0}$ & 10.42 & $8.34 / 1.9673 \mathrm{e}-4$ & $8.34 / 1.9673 \mathrm{e}-4$ & $8.18 / 1.9714 \mathrm{e}-4$ & 0.2 \\
\hline $\mathbf{3 0 0}$ & 10.81 & $8.65 / 3.7619 \mathrm{e}-4$ & $8.65 / 3.7619 \mathrm{e}-4$ & $8.46 / 3.7721 \mathrm{e}-4$ & 0.27 \\
\hline $\mathbf{6 0 0}$ & 11.2 & $8.96 / 7.3203 \mathrm{e}-4$ & $8.96 / 7.3203 \mathrm{e}-4$ & $8.68 / 7.3567 \mathrm{e}-4$ & 0.5 \\
\hline $\mathbf{9 0 0}$ & 11.42 & $9.14 / 1.0744 \mathrm{e}-3$ & $8.57 / 1.0843 \mathrm{e}-3$ & $8.76 / 1.0835 \mathrm{e}-3$ & 0.08 \\
\hline $\mathbf{1 2 0 0}$ & 11.64 & $9.31 / 1.4154 \mathrm{e}-3$ & $8.73 / 1.4448 \mathrm{e}-3$ & $8.79 / 1.4399 \mathrm{e}-3$ & 0.34 \\
\hline $\mathbf{1 5 0 0}$ & 11.75 & $9.40 / 1.7484 \mathrm{e}-3$ & $8.81 / 1.8048 \mathrm{e}-3$ & $8.75 / 1.7961 \mathrm{e}-3$ & 0.48 \\
\hline $\mathbf{4 5 0}$ & 11.12 & $8.90 / 5.4355 \mathrm{e}-4$ & $8.34 / 5.4460 \mathrm{e}-4$ & $8.65 / 5.4586 \mathrm{e}-4$ & 0.23 \\
\hline $\mathbf{7 5 0}$ & 11.39 & $9.11 / 9.0118 \mathrm{e}-4$ & $8.54 / 9.1319 \mathrm{e}-4$ & $8.77 / 9.0819 \mathrm{e}-4$ & 0.55 \\
\hline
\end{tabular}

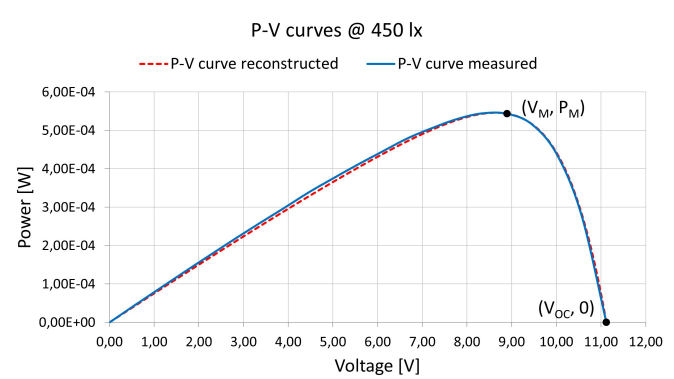

Fig. 8. Measured vs reconstructed P-V curve at $450 \mathrm{~lx} .\left(V_{M}, P_{M}\right)$ and $\left(V_{O C}, 0\right)$ sampled when $\mathrm{k}=0.8$. 


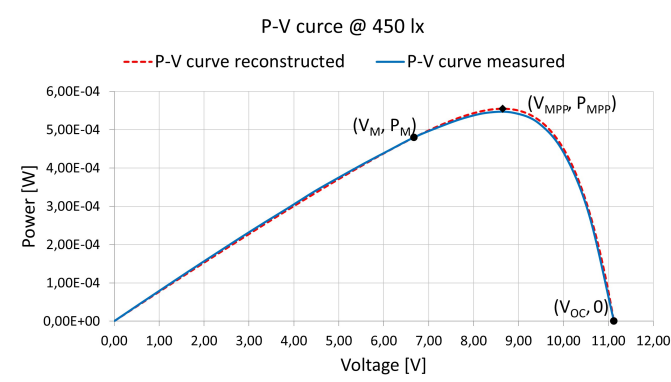

Fig. 9. Measured vs reconstructed P-V curve at $450 \mathrm{~lx} .\left(V_{M}, P_{M}\right)$ and $\left(V_{O C}, 0\right)$ sampled when $\mathrm{k}=0.6$.

\section{Application of the Procedure to Verify FOCV-MPPT Efficiency}

The previous curves are reconstructed starting from $\left(V_{M}\right.$, $P_{M}$ ) measured when $k$ is programmed at 0.8 : observing from Table I and Fig. 7-8 how low the error is between $\left(V_{M}, P_{M}\right)$ and $\left(V_{M P P}, P_{M P P}\right)$, it can be concluded that the FOCV multiplying factor $k=0.8$ is near the optimum value for the prototype system. A second set of curves is reconstructed from measurements taken when programming the dc-dc converter at $k=0.6$, as could be suggested from typical values in the Sanyo AM-1801 datasheet (see section IV.B). As an example, Fig. 9 shows the reconstructed curve at 450 lx; it can be observed that even if the operating point in this case is far away from the MPP, the reconstructed curve is still a good approximation of the measured one. The error in the estimated $P_{M P P}$ is larger than in the previous cases but less than $2 \%$ while the $V_{M P P}$ difference is still in the same order $(0.3 \mathrm{~V})$. It can easily be observed that the operating point $V_{M}$ is lower than the optimum one $V_{M P P}$, which enables one to state that $k=V_{M} / V_{O C}$ is lower than optimum and suggests the correcting action of increasing $k$ by acting on the voltage divider at the dc-dc converter input. Similar results were obtained for different illuminance levels and programmed $k$ values, showing that the proposed technique allows an accurate estimate of MPPT efficiency to be made without requiring a scan of the full PV panel characteristic.

\section{CONCLUSIONS}

The paper presents an algorithm to reconstruct the P-V characteristic of an indoor PV cell without measuring the shortcircuit current but only the open-circuit voltage and a generic point on the curve near the MPP. This feature allows one to reconstruct the characteristic when the PV cell is integrated in a harvesting system composed of the cell itself and a dc-dc converter implementing an FOCV-MPPT technique without additional dedicated power-consuming circuitry or power loss due to short-circuit current measuring. The algorithm combined with an external measurement unit, connected during the debug phase in series between the transducer and the dc-dc converter, is applied so as to verify the efficiency of the MPP tracking technique in a prototype energy harvesting system composed of indoor PV panels. Experimental results enable the error between the measured and reconstructed curves in the MPP point to be evaluated; the $P_{M P P}$ error is low (less than $1 \%$ ) if the measured point used to reconstruct the curve is near the MPP. This error increases (less than $2 \%$ ) if it is far away from the MPP, but the difference in the estimated $V_{M P P}$ remains in the same range $(0.3 \mathrm{~V})$. The difference between the calculated $V_{M P P}$ and the transducer operating point $V_{M}$ is a clear indicator if the $k$ factor is not optimum for maximizing power transfer; by observing the reconstructed characteristic, one can easily understand the corrective action to be taken on the multiplying $k$ factor.

\section{ACKNOWLEDGMENT}

This project has received funding from the Electronic Component Systems for European Leadership Joint Undertaking under grant agreement No 737434. This Joint Undertaking receives support from the European Union's Horizon 2020 research and innovation programme and Germany, Slovakia, Netherlands, Spain, Italy.

\section{REFERENCES}

[1] B. Subudhi and R. Pradham, "A comparative study on maximum power point tracking techniques for photovoltaic power system", IEEE Trans. on Sustainable Energy, vol. 4. No. 1, pp. 89-98, Jan. 2013.

[2] J. Ahmad, "A fractional open circuit voltage based maximum power point tracker for photovoltaic arrays", in Proc. 2nd Int. Conf. Softw. Technol. Eng., Oct. 2010, pp. 247-250.

[3] M. A. S. Masoum, H. Dehbonei and E. F. Fuchs, "Theoretical and experimental analyses of photovoltaic systems with voltageand currentbased maximum power-point tracking," in IEEE Transactions on Energy Conversion, vol. 17, no. 4, pp. 514-522, Dec 2002.

[4] C. Rodriguez and G. A.J. Amaratunga, "Analytic solution to the photovoltaic maximum power point problem", IEEE Trans. on Circuits and Systems-I, vol. 54, no. 9 pp. 2054-2060, Sept. 2007.

[5] B. Subudhi and R. Pradhan, "Characteristics evaluation and parameter extraction of a solar array based on experimental analysis," 2011 IEEE Ninth International Conference on Power Electronics and Drive Systems, Singapore, 2011, pp. 340-344.

[6] Y. Wang et al., "Storage-less and converter-less photovoltaic energy harvesting with maximum power point tracking for Internet of Things," in IEEE Transactions on Computer-Aided Design of Integrated Circuits and Systems, vol. 35, no. 2, pp. 173-186, Feb. 2016.

[7] A. Virtuani, E. Lotter, M. Powalla, "Influence of the light source on the low-irradiance performance of $\mathrm{Cu}(\mathrm{In}, \mathrm{Ga}) \mathrm{Se} 2$ solar cells", Solar Energy Materials and Solar Cells, vol. 90, issue 14, pp. 2141-2149, Sept 2006.

[8] A. Montecucco and A. R. Knox, "Maximum power point tracking converter based on the open-circuit voltage method for thermoelectric generators," in IEEE Transactions on Power Electronics, vol. 30, no. 2, pp. 828-839, Feb. 2015

[9] J. Merten, J. M. Asensi, C. Voz, A. V. Shah, R. Platz and J. Andreu, "Improved equivalent circuit and analytical model for amorphous silicon solar cells and modules", IEEE Trans. on Electron Devices, vol. 45, no. 2, pp. 423-429, Feb. 1998.

[10] D. Sera, R. Teodorescu and P. Rodriguez, "PV panel model based on datasheet values," 2007 IEEE International Symposium on Industrial Electronics, Vigo, Spain, 2007, pp. 2392-2396.

[11] J. C. Lagarias, J. A. Reeds, M. H. Wright and P. Wright, "Convergence properties of the Nelder-Mead simplex method in low dimensions", SIAM Journal of Optimization, Vol. 9 Number 1, pp. 112-147, 1998.

[12] J. C. H. Phang, D. S. H. Chan and J. R. Phillips, "Accurate analytical method for the extraction of solar cell model parameters," in Electronics Letters, vol. 20, no. 10, pp. 406-408, May 101984.

[13] C. T. Kelley, Solving nonlinear equations with Newton's Method. Siam, 2003, p. 112.

[14] Sanyo AM-1801 datasheet [Online].

[15] STMicroelectronics SPV1050 datasheet [Online]. 